\title{
The core genome multi-locus sequence typing of Mycoplasma anserisalpingitidis
}

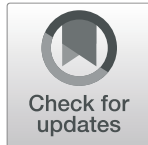

Áron B. Kovács ${ }^{1 \dagger}$, Zsuzsa Kreizinger ${ }^{1 \dagger}$, Barbara Forró ${ }^{1 \dagger}$, Dénes Grózner ${ }^{1}$, Alexa Mitter ${ }^{1}$, Szilvia Marton ${ }^{1}$, Krisztina Bali $^{1}$, Anna Sawicka², Grzegorz Tomczyk², Krisztián Bányai ${ }^{1}$ and Miklós Gyuranecz ${ }^{1,3^{*}}$ (D)

\begin{abstract}
Background: Mycoplasma anserisalpingitidis is a waterfowl pathogen that mainly infects geese, can cause significant economic losses and is present worldwide. With the advance of whole genome sequencing technologies, new methods are available for the researchers; one emerging methodology is the core genome Multi-Locus Sequence Typing (cgMLST). The core genome contains a high percentage of the coding DNA sequence (CDS) set of the studied strains. The cgMLST schemas are powerful genotyping tools allowing for the investigation of potential epidemics, and precise and reliable classification of the strains. Although whole genome sequences of M. anserisalpingitidis strains are available, to date, no cgMLST schema has been published for this species.

Results: In this study, Illumina short reads of 81 M. anserisalpingitidis strains were used, including samples from Hungary, Poland, Sweden, and China. Draft genomes were assembled with the SPAdes software and analysed with the online available chewBBACA program. User made modifications in the program enabled analysis of mycoplasmas and provided similar results as the conventional SeqSphere+ software. The threshold of the presence of CDS in the strains was set to $93 \%$ due to the quality of the draft genomes, resulting in the most accurate and robust schema. Three hundred thirty-one CDSs constituted our cgMLST schema (representing 42,77\% of the whole CDS set of M. anserisalpingitidis ATCC BAA-2147), and a Neighbor joining tree was created using the allelic profiles. The correlation was observed between the strains' cgMLST profile and geographical origin; however, strains from the same integration but different locations also showed close relationship. Strains isolated from different tissue samples of the same animal revealed highly similar cgMLST profiles.

Conclusions: The Neighbor joining tree from the cgMLST schema closely resembled the real-life spatial and temporal relationships of the strains. The incongruences between background data and the CgMLST profile in the strains from the same integration can be because of the higher probability of contacts between the flocks. This schema can help with the epidemiological investigation and can be used as a basis for further studies.
\end{abstract}

Keywords: cgMLST, chewBBACA, Genotyping, Mycoplasma anserisalpingitidis, Waterfowl, Whole genome sequencing

\footnotetext{
* Correspondence: m.gyuranecz@gmail.com

†Áron B. Kovács, Zsuzsa Kreizinger and Barbara Forró contributed equally to this work.

${ }^{1}$ Institute for Veterinary Medical Research, Centre for Agricultural Research, Hungária krt 21, Budapest H-1143, Hungary

${ }^{3}$ Department of Microbiology and Infectious Diseases, University of Veterinary Medicine, H-1078 István utca 2, Budapest, Hungary

Full list of author information is available at the end of the article
}

(c) The Author(s). 2020 Open Access This article is licensed under a Creative Commons Attribution 4.0 International License, which permits use, sharing, adaptation, distribution and reproduction in any medium or format, as long as you give appropriate credit to the original author(s) and the source, provide a link to the Creative Commons licence, and indicate if changes were made. The images or other third party material in this article are included in the article's Creative Commons licence, unless indicated otherwise in a credit line to the material. If material is not included in the article's Creative Commons licence and your intended use is not permitted by statutory regulation or exceeds the permitted use, you will need to obtain permission directly from the copyright holder. To view a copy of this licence, visit http://creativecommons.org/licenses/by/4.0/. The Creative Commons Public Domain Dedication waiver (http://creativecommons.org/publicdomain/zero/1.0/) applies to the data made available in this article, unless otherwise stated in a credit line to the data. 


\section{Background}

Mycoplasma anserisalpingitidis is a waterfowl pathogenic bacterium that was first isolated in 1983 in Hungary [1, 2]. It commonly occurs alongside $M$. anseris, M. cloacale, and M. anatis and is present all over the world. It can be isolated from geese and occasionally from ducks [3]. This species can be transmitted both vertically and horizontally $[4,5]$. The pathogen can cause significant economic losses [6-8]. Symptoms can include phallus and cloaca inflammation, egg infertility, airsacculitis, salpingitis, peritonitis, and embryo lethality [4-6]. In recent years the investigation of these bacteria have become a research priority [9]. In 2019 a speciesspecific PCR system was designed [3] that led to improved laboratory diagnosis. No vaccine is available for this mycoplasma as of yet, but antibiotics are used routinely to treat the infection [10]. For these reasons, the epidemiological monitoring and study of $M$. anserisalpingitidis is of high importance.

Conventional MLST system [11, 12] is among the most widespread molecular typing method, which focuses on the examination of 5 to 7 house-keeping genes, that can be found in all investigated strains. While this small selection allows for the sequencing of the genes even in bacteria, from which whole genome sequences are not possible to acquire, it still lacks the discriminatory power of the gene-by-gene comparison methods. In contrast, the core genome (cg) MLST system uses all the coding sequences that can be found in the majority of the strains. This is the reason why cgMLST schemas have higher resolution and are less susceptible to deletions and other mutations in the genomes, providing a more robust analysis than single nucleotide polymorphisms (SNP) based methods. With the recent advances in whole genome sequencing and a significant reduction of per-base costs, it is now easier to determine quickly and reliably the whole genome sequence and with it have access to a wider array of data than before. There are a few commercial software packages that can be purchased for cgMLST studies, for example, Ridom SeqSphere+ [13] and Bionumerics [14]. In recent years two cgMLST schemas have been developed for avian mycoplasmas using one of these platforms $[15,16]$. Besides, open-source options are available, such as chewBBACA, which has been successfully used in many cgMLST studies for a multitude of microorganisms since its release [17-19].

Mycoplasma anserisalpingitidis has only recently been fully characterized [20], and as of the writing of this study, no cgMLST schema exists for M. anserisalpingitidis. The aim of the present examination was the genetic characterisation of $81 \mathrm{M}$. anserisalpingitidis strains with a robust and reliable cgMLST schema created in this study.

\section{Results}

Whole genome assembly

A total of $81 \mathrm{M}$. anserisalpingitidis whole genome sequences were used in this study, resulting in 81 multi fasta files with the average longest contig being 96,619 bp. Sequence reads of strains ATCC BAA-2147, MYCAV93, and MYCAV177 are available under the BioProject number: PRJNA554588, PRJNA553666, PRJNA554567 respectively. The raw nucleotide sequence reads of the further $78 \mathrm{M}$. anserisalpingitidis were uploaded to the Sequence Read Archive (SRA) of NCBI under the BioProject numbers: PRJNA602215, PRJNA602206, and PRJNA603657.

\section{Development of $M$. anserisalpingitidis cgMLST cgMLST schema selection and evaluation}

Using the modified chewBBACA algorithm a total of 1272 loci were selected for the wgMLST schema. Out of these loci, four cgMLST schemas were extracted based on different CDS presence thresholds (100, 95, 93, and $90 \%$ presence). Out of the examined four CDS presence thresholds, the 93\% (including $331 \mathrm{CDSs}$ ) one resembled the metadata the most. The 331 loci represent approximately $42.77 \%$ of the whole CDS set of $M$. anserisalpingitidis ATCC BAA-2147. The most conservative allele showed six variants (MYCAV429-protein75), while 67 different genotypes were determined on the most variable allele (MYCAV429-protein17) among the $81 \mathrm{M}$. anserisalpingitidis strains.

Phylogenetic trees created from the initial wgMLST schema (data not shown) and the one from the final cgMLST schema showed similar topologies. Strains originating from the known same integration (Supplementary Table 1) showed close relationship and belonged to cluster I, while strains from other integrations and countries formed cluster II (Fig. 1).

In cluster I branch lengths between the subclades were notably shorter, than in cluster II, and cgMLST profiles did not correspond to geographical origins; however, subclades were formed by strains isolated mostly in the same year or in following years (Fig. 1). It should be noted, that three strains were included in cluster I, which originated from farms that did not belong to the integration forming the cluster. These strains were MYCAV47, the strain isolated from a duck, originating from Tazlar, approximately $80 \mathrm{~km}$ far from the closest farm (Szentes) of the integration; MYCAV49, a strain from Tiszavasvari, approximately $50 \mathrm{~km}$ far from the closest farm (Erpatak) of the integration and MYCAV69, a strain from Ludas, approximately $70 \mathrm{~km}$ far from the closest farm (Cered) of the integration.

A correlation was observed between the strains' cgMLST profile and geographical origin in cluster II, in most cases strains from the same country (i.e. Poland) or 


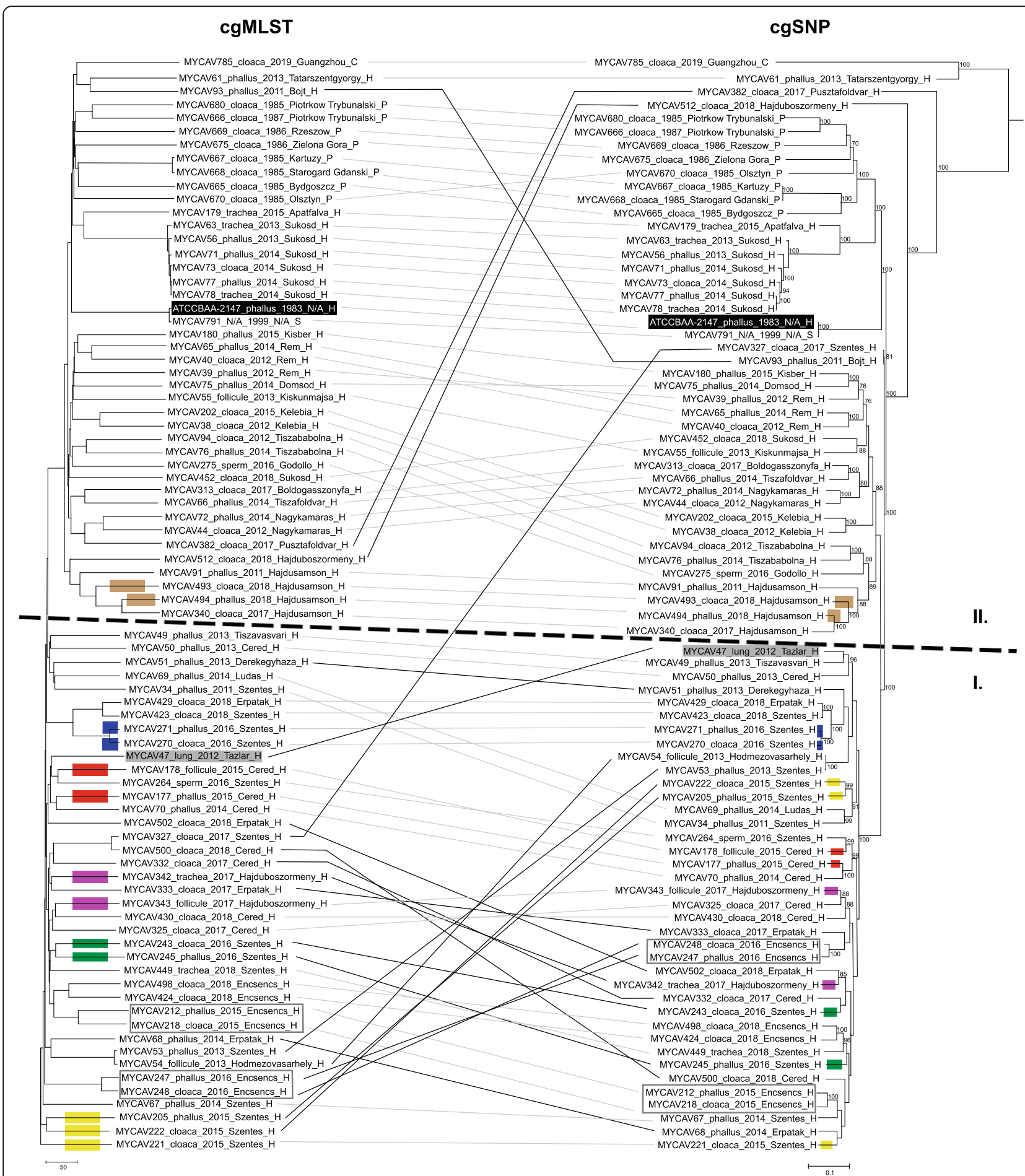

Fig. $1 \mathrm{cgMLST}$ and cgSNP based phylogenetic trees of 81 M. anserisalpingitidis strains. Strain ID, tissue sampled, year of isolation, location of origin, and country of origin are given for each strain. Abbreviations: lung - lung and air sac, phallus - phallus lymph, C - China, H - Hungary, P - Poland, S - Sweden, N/A - not available. The M. anserisalpingitidis type strain ATCC BAA-2147 is highlighted in black. Strains originating from the same animal are in grey rectangles. Coloured rectangles on the branches indicate that the strains originated from the same flocks, each colour represents a separate flock. Identical strains with similar or notably different topologies on the two dendrograms are bound with grey or black lines, respectively. cgMLST. Neighbor joining tree based on M. anserisalpingitidis cgMLST allelic profiles determined by the modified chewBACCA software. The dendrogram was created with GrapeTree software version 1.5 .0 [21] cgSNP. Maximum Likelihood tree based on core genome SNP analysis. The dendrogram was created using the General Time Reversible model with Gamma distribution and bootstrap set to 500 iterations in MEGA-X version 10.0.5 [22]. Bootstrap values $\geq 70$ are presented 
same farms formed separate subclades (strains from Hungary, e.g. MYCAV91, MYCAV340, MYCAV493, and MYCAV494 are from the same city while MYCAV512 is from a city only approximately $20 \mathrm{~km}$ away). Strains from Sukosd, Hungary (the farm with the highest number of isolated strains in cluster II; $n=7$ ) showed a correlation between year of isolation and their cgMLST profile also, forming two separate subclades (MYCAV452 from 2018 and MYCAV56, 63, 71, 73, 77 and MYCAV78 from 2013 and 2014) (Fig. 1).

Strains isolated from different tissue samples of the same animal (MYCAV212 and MYCAV218; MYCAV247 and MYCAV248) revealed highly similar cgMLST profiles. The majority of samples from the same flock also appear on the same branch (e.g. MYCAV205, MYCAV221 and MYCAV222 or MYCAV243, MYCAV245). However, example for strains from the same flocks with greater genetic distance between them (MYCAV177 and MYCAV178, MYCAV342 and MYCAV343) was observed as well. In these cases, the strains originated from different tissue samples of the birds (Fig. 1).

\section{Comparison of the developed cgMLST schema with core genome SNP based analysis}

The DNASTAR LaserGene suite found 25,518 SNPs in the pseudo-genome. The Maximum Likelihood phylogenetic tree based on the SNPs of the cgMLST CDS set and the Neighbor Joining tree from the cgMLST schema gave highly similar results. The three strain that originated from farms that did not belong to the integration forming cluster I remained in cluster I with the cgSNP analysis as well. The cgSNP based method also clustered most of the samples from the same flocks or animals together (e.g. MYCAV212 and MYCAV218, MYCAV245 and MYCAV243 or MYCAV 248 and 247); however, in certain cases the cgSNP based method was less sensitive (e.g. MYCAV205, MYCAV221 and MYCAV222 or MYCAV243 and MYCAV245). Notable differences were mainly observed in the topology of cluster I, and one strain (MYCAV327) belonging to the integration from where strains in cluster I originated was placed in cluster II in the cgSNP based dendrogram (Fig. 1).

\section{Discussion}

Mycoplasma anserisalpingitidis is a common waterfowl pathogen present worldwide [2, 23]. Despite the economic losses it can cause, there are still major gaps in our knowledge about this microorganism. There is no vaccine for $M$. anserisalpingitidis as of yet and mainly antibiotic therapy can be used for the treatment of infected flocks and the fact that antibiotic resistance shows a growing trend among pathogenic microbes [10], warrants the inspection and monitoring of this bacterium.
As a molecular biology based monitoring method, the cgMLST focuses on a large selection of CDSs found in most of the strains in a study, allowing high discriminatory power and making the system less susceptible to mutations, such as insertions and deletions.

The present study describes the first cgMLST schema for $M$. anserisalpingitidis. The open- source software BSR-Based Allele Calling Algorithm (chewBBACA) [24], adjusted with the consent of the original developers to be compatible with the mycoplasma genetic code (genetic code 4), was used to create the cgMLST schema. The modified algorithm provided highly similar results during the verification to previously published analyses on M. gallisepticum [15]. The developed M. anserisalpingitidis cgMLST assay constituted of 331 CDSs, $42.77 \%$ of the CDS set of $M$. anserisalpingitidis type strain ATCC BAA-2147. The CDSs in the schema were present in more than $93 \%$ of the strains in this study, which is a more lenient criterion than the default $100 \%$ in chewBBACA or the commonly used 95\% [24], but it made for a more precise and robust assay. The suitability of the lower threshold value can be explained by the possible higher mutation rate of mycoplasmas [25, 26] or the quality of the sequencing and assembly. Deeper sequencing can improve the outcome, but it is important to note that our schema gave a precise result, which correlated with the epidemiological data, thus such deeper sequencing was not deemed necessary. Using this schema resulted in an assay that managed to differentiate between all of the strains in this study and group them into sub-clades that were continuous with the metadata of the samples.

The cgMLST analysis based genetic relations among the $M$. anserisalpingitidis strains were presented on Neighbor joining tree from the cgMLST profiles determined by chewBBACA, not on the sequences per se, which limits the usable topology metrics in setting up a phylogenetic tree. Nevertheless, the wgMLST and cgMLST based phylogenetic analyses showed high similarity, which indicates that the core genome schema is robust enough to be used as a basis of further studies, and the fact that the geographically and temporally close samples tend to be in the same groups further proves its precision.

The majority of the samples in this study originated from Hungary. While this fact restricts the scope of this assay, it also shows that the resolution of the method is exceptional, as in most cases even the strains with relatively small geographical distances are separated on the Neighbor joining tree. The fact that the strains from the same integration (cluster I, Fig. 1) showed higher variability and lacked correlation between genotype and geographical origin could indicate that the hosts were moved frequently or had contact with each other. This practice could hinder the defence against the bacterium 
as a potentially pathogen free stock could be infected or re-infected.

The results of the developed cgMLST schema and cgSNP based analysis were highly congruent, with notable differences in the topology observed mostly among the strains in cluster I (Fig. 1). The reason behind the observed differences can be the fact that the two software used for the analyses (chewBBACA and LaserGene) apply different approaches to find SNPs (CDS alignment and read mapping, respectively). It is also important to note that the cgSNP based phylogenetic tree was assessed with a different method (Maximum Likelihood) than the allelic profile based tree (Neighbor joining).

The developed cgMLST assay proved to be robust and showed high resolution, revealing high variability of the examined $81 M$. anserisalpingitidis strains, and discriminating two main clusters among them congruent with the strains' epidemiologic data (Fig. 1). The fact that the assay had to be preceded by time-consuming whole genome sequencing is greatly offset by the lack of primer designing and validating steps. Besides, as the target species is a relatively fast growing bacterium, with lower requirements during isolation compared to other Mycoplasma species, the presented cgMLST can be recommended for diagnostic use also.

The relatively high number of Hungarian samples indicates that the infection may be widespread in the Carpathian Basin, but the fact that there were samples from Poland, Sweden, and China suggests that the microorganism can be found all over the world. China is the world leader in goose production by a huge margin and it is especially important to further explore the extent of $M$. anserisalpingitidis infection there [27]. It is important to note that the lack of a substantial number of samples from different countries is likely because of the relatively small awareness of this particular pathogen instead of the lack of infection in waterfowl stocks. Our cgMLST assay allows for the precise typing of $M$. anserisalpingitidis strains, which can lead to a better understanding and defence against this pathogen.

\section{Conclusions}

The presented cgMLST schema is the first published genotyping assay to differentiate between $M$. anserisalpingitidis strains from different integrations, geographical location, and time period. The high resolution of the developed cgMLST allows its use in phylogenetic analyses and diagnostics of potential outbreaks, and it can be the basis of future studies exploring the phenotypic differences of the strains in different subclades.

\section{Methods}

Strain preparation and whole genome assembly

A total of $78 \mathrm{M}$. anserisalpingitidis genomes were sequenced successfully in this study originating from
Hungary $(n=68)$, Poland $(n=8)$, Sweden $(n=1)$ and China $(n=1)$. The detailed information of the strains can be found in Supplementary Table 1. DNA was extracted from the strains with the QIAamp DNA Mini kit (Qiagen, Inc., Hilden, Germany). Next-generation sequencing was performed on NextSeq 500 Illumina equipment (Illumina, Inc., San Diego, CA USA), with NextSeq 500/550 High Output Kit v2.5 reagent kit. Publicly available whole genome sequences of an additional three strains, ATCC BAA-2147, MYCAV93, and MYCAV177 [9] were involved also in the analyses, resulting in 81 strains altogether. The raw sequence reads were first quality checked with the FastQC software version 0.11.8 (downloaded on 2019.02.13) [28], and trimmed with the NxTrimm software version 0.4.3 (downloaded on 2019.02.13) [29] and paired with Geneious Prime version 2019.2.1 (downloaded on 2018.11.12) [30]. The draft genomes were assembled with the SPAdes program version 3.13.0 (downloaded on 2019.03.13) [31].

\section{Adjustment and verification of chewBBACA software for mycoplasma genetic code compatibility}

The BSR-Based Allele Calling Algorithm (chewBBACA) version 2.0.17 (downloaded on 2019.06.22) [24] was used to create a whole-genome $(\mathrm{wg})$ and then a core genome (cg) schema. As of the writing of this article, chewBBACA is hard coded to only use the standard genetic code 11 which creates an obstacle in analysing any bacteria using genetic code 4 (the mycoplasma genetic code). For this reason, the source code had to be modified slightly to serve as an adequate framework for mycoplasmas. The only difference between the standard genetic code (11) and mycoplasma genetic code (4) is that the TGA codon codes Tryptophan (Trp) instead of a stop codon [32]. Hence, every instance in the chewBBACA source code referring to genetic code 11 was changed to genetic code 4 , but the software was not tampered with besides this small change, which was done with the knowledge and approval of the original software developers. The chewBBACCA software makes use of the Prodigal program version 2.6.0 (downloaded on 2019.06.22) or above [33] for finding Coding DNA Sequence (CDS) in the whole or draft genomes and BLAST + version 2.9.0 (downloaded on 2019.06.22) [34] for comparison between the alleles.

As a first step in the verification of the modified software, data from the whole genome fasta file of $M$. anserisalpingitidis ATCC BAA-2147 annotated with Prodigal were compared to the NCBI annotated genome. The Prodigal managed to find all of the CDSs in the sequence with the exception of 11 .

The applicability of the modified software was further checked using a previously developed M. gallisepticum 
cgMLST schema [15] as a comparison. The sequence reads of $M$. gallisepticum strains from BioProject PRJNA401291 were downloaded and assembled into draft genomes. The 55 assembled draft genomes were then analysed with chewBBACA as described below. The allelic profiles of the Ridom SeqSphere+ and chewBACCA cgMLST schemas (Supplementary Table 2) were used in the creation of two Neighbor joining trees with GrapeTree software version 1.5.0 (downloaded on 2020.04.) [21] using the FastME implementation [35] as seen in Supplementary Fig. 1.

\section{Development of $M$. anserisalpingitidis cgMLST}

The creation of the $M$. anserisalpingitidis cgMLST schema was done with the modified chewBBACA version 2.0.17 [24]. First, a wgMLST schema was set up containing every CDS, and then the paralogous alleles were filtered, thus creating the cgMLST schema.

\section{Creation of wgMLST}

The wgMLST schema of $M$. anserisalpingitidis was created with the modified chewBBACA software [24]. A training file was created in Prodigal [33] with $M$. anserisalpingitidis ATCC BAA-2147 and this file was used in the further steps of the study. The wgMLST schema from the $81 M$. anserisalpingitidis strains was assessed first with the CreateSchema operation, during which every genome is annotated one by one. Sequences are compared in a pairwise fashion and an all-against-all BLASTP search and the BLAST score ratio (BSR) is calculated [36]. This allows for the genes that are coding the same or very similar (by default BSR above 0.6) proteins to be considered alleles of the same locus and to be collected in a database. The second step of the wgMLST creation is the AlleleCall operation during which the software populates the schema and an algorithm detects whether a CDS can correspond to more than one locus and a BLASTP database is created from all the translated CDSs. This database can be used as a base for the BLASTP search, during which the sequence can be classified either as a new locus or as a missing one.

\section{Extraction of the cgMLST schema}

The quality of the wgMLST schema was tested with the TestGenomeQuality operation. During the quality test, it was found that the majority of the CDSs were not present in all genomes. Four cgMLST schemas were extracted from the wgMLST schema with the ExtractCgMLST operation of the modified chewBBACA software, with CDS presence thresholds 100, 95, 93, and $90 \%$. Neighbor-joining trees were created from the extracted allelic profiles in Phyloviz software version 2.0 (downloaded on 2019.09.12) using Hamming distance and Saitou - Nei criterion [37, 38] (data not shown). With the $100 \%$ presence rate, the schema consisted of only 73 CDSs, while 291 with $95 \%, 331$ with $93 \%$ and 408 CDSs were present with the $90 \%$ threshold. The results of the four cgMLST schemas were manually examined and the one best representing the metadata of the strains was chosen. The best result was achieved with a 93\% threshold with 331 CDSs that were present in the schema, constituting of approximately $42.77 \%$ of the CDS set of $M$. anserisalpingitidis ATCC BAA-2147.

\section{Comparison of the developed cgMLST schema with core genome SNP based analysis}

The CDSs constituting our cgMLST schema were extracted from the strain MYCAV93, randomly chosen out of the available whole genomes on NCBI. These CDSs were concatenated, creating a pseudo-genome. The sequence reads of the 80 strains (all examined strains except MYCAV93) were mapped with DNASTAR LaserGene suite version 17.0.2.1 (downloaded on 2020.04.20) [39]. The SNPs were filtered and an alignment was created from the ones corresponding to the same position. The alignment was analysed with MEGA-X version 10.0.5. (downloaded on 2019.02.25) [22] and a Maximum Likelihood tree was created using the General Time Reversible model with Gamma distribution and bootstrap set to 500 iterations. The cgSNP analysis based phylogenetic tree was compared with the Neighbor joining tree based on cgMLST profiles assessed by GrapeTree software version 1.5.0 (Fig. 1).

\section{Supplementary information}

Supplementary information accompanies this paper at https://doi.org/10. 1186/s12864-020-06817-2.

Additional file 1: Supplementary Table 1. Background information and cgMLST profiles of the $81 \mathrm{M}$. anserisalpingitidis strains examined in the study.

Additional file 2: Supplementary Table 2. chewBBACA cgMLST profiles of $55 \mathrm{M}$. gallisepticum strains analysed with Ridom SeqSphere+ in a previous study. The cgMLST profiles of $M$. gallisepticum strains from the publication of Ghanem et al. [15] were determined with the in-house adjusted chewBBACA software [24], compatible with the mycoplasma genetic code.

Additional file 3: Supplementary Figure 1. Comparison between two M. gallisepticum phylogenetic trees using the Ridom SeqSphere+ and chewBBACA cgMLST schemas. Neighbor joining trees were created with GrapeTree software version 1.5.0 [21]. Identical strains with similar or notably different topologies on the two dendrograms are bound with grey or black lines, respectively. A. Phylogenetic tree created based on M. gallisepticum cgMLST allelic profiles determined by Ridom SeqSphere+ software [15]. B. Phylogenetic tree created based on M. gallisepticum cgMLST allelic profiles determined by the modified chewBACCA software.

\section{Abbreviations}

MLST: Multi-Locus Sequence Typing; wgMLST: Whole genome Multi-Locus Sequence Typing; cgMLST: Core genome Multi-Locus Sequence Typing; CDS: Coding DNA sequence; chewBBACA: BSR-Based Allele Calling 
Algorithm; NCBI: National Center for Biotechnology Information; BSR: BLAST score ratio; SRA: Sequence Read Archive

\section{Acknowledgments}

The authors would like to thank Janet Bradbury and Christopher J. Morrow for their help in the acquisition of Swedish and Chinese M. anserisalpingitidis strains.

\section{Authors' contributions}

ÁBK and MG designed the study. ÁBK analysed the sequences and data, as well as wrote the manuscript. ZK analysed the data and wrote the manuscript. BF, SM, KB1, and KB2 performed the whole genome sequencing, analysed the sequences, and edited the manuscript. DG, AM, AS, GT and MG processed the samples, analysed the data, and edited the manuscript. All authors read and approved the final version of the manuscript.

\section{Funding}

This work was supported by the Lendület program (LP2012-22) of the Hungarian Academy of Sciences, the K_16 (119594), and KKP19 (129751) grants of the National Research, Development and Innovation Office, Hungary. ZK and MG were supported by the Bolyai János Fellowship of the Hungarian Academy of Sciences and by the Bolyai+ Fellowship (ÚNKP-19-4ATE-1) of the New National Excellence Program of the Ministry of Innovation and Technology. The funders had no role in study design, data collection, and interpretation, or the decision to submit the work for publication.

\section{Availability of data and materials}

All data generated or analysed during this study are included in this published article. The sequence reads of the strains have been uploaded to the Sequence Read Archive (SRA) under: PRJNA554588, PRJNA553666, PRJNA554567, PRJNA602215, PRJNA602206, and PRJNA603657. The cgMLST schema can be found under: https://doi.org/10.6084/m9. figshare.12129054

\section{Ethics approval and consent to participate}

According to the written declaration (reference number: IVMR/2019/0005) of the Ethics Committee of the Institute for Veterinary Medical Research, Centre for Agricultural Research ethical approval was not required for the study as the samples were taken during routine diagnostic examinations with the written consent of the owners. The final study and the manuscript was submitted and approved in a written declaration by the Ethics Committee of the Institute for Veterinary Medical Research, Centre for Agricultural Research.

\section{Consent for publication}

Not applicable.

\section{Competing interests}

The authors declare that they have no competing interests.

\section{Author details}

'Institute for Veterinary Medical Research, Centre for Agricultural Research, Hungária krt 21, Budapest H-1143, Hungary. ${ }^{2}$ Department of Poultry Diseases, National Veterinary Research Institute, Aleja Partyzantow 57, 24-100 Pulawy, Poland. ${ }^{3}$ Department of Microbiology and Infectious Diseases, University of Veterinary Medicine, H-1078 István utca 2, Budapest, Hungary.

\section{Received: 3 March 2020 Accepted: 9 June 2020}

Published online: 15 June 2020

\section{References}

1. Stipkovits L, Varga Z, Dobos-Kovacs M, Santha M. Biochemical and serological examination of some mycoplasma strains of goose origin. Acta Vet Hung. 1984;32:117-25.

2. Volokhov D V., Grózner D, Gyuranecz M, Ferguson-Noel N, Gao Y, Bradbury $J M$, et al. Mycoplasma anserisalpingitidis sp. nov., isolated from European domestic geese (Anser anser domesticus) with reproductive pathology. Int J Syst Evol Microbiol. 2020;:ijsem004052. doi:https://doi.org/10.1099/ijsem.0. 004052.

3. Grózner D, Sulyok KM, Kreizinger Z, Rónai Z, Jánosi S, Turcsányi I, et al. Detection of mycoplasma anatis, M. anseris, M. cloacale and mycoplasma sp. 1220 in waterfowl using species-specific PCR assays. PLoS One. 2019;14.
4. Stipkovits L, Varga Z, Glávits R, Rátz F, Molnár E. Pathological and immunological studies on goose embryos and one-day-old goslings experimentally infected with a mycoplasma strain of goose origin. Avian Pathol. 1987;16:453-68.

5. Stipkovits L, Varga Z, Czifra G, Dobos-Kovacs M. Occurrence of mycoplasmas in geese affected with inflammation of the cloaca and phallus. Avian Pathol. 1986;15:289-99.

6. Stipkovits L, Kempf I. Mycoplasmoses in poultry. Rev Sci Tech. 1996;15:1495-525.

7. Stipkovits L, Szathmary S. Mycoplasma infection of ducks and geese. Poult Sci. 2012;91:2812-9.

8. Dobos-Kovács M, Varga Z, Czifra G, Stipkovits L. Salpingitis in geese associated with mycoplasma sp. strain 1220. Avian Pathol. 2009:38:239-43.

9. Grózner D, Forró B, Kovács ÁB, Marton S, Bányai K, Kreizinger Z, et al. Complete Genome Sequences of Three Mycoplasma anserisalpingitis (Mycoplasma sp. 1220) Strains. Microbiol Resour Announc. 2019;8.

10. Grózner D, Kreizinger Z, Sulyok KM, Rónai Z, Hrivnák V, Turcsányi I, et al. Antibiotic susceptibility profiles of mycoplasma sp. 1220 strains isolated from geese in Hungary. BMC Vet Res. 2016;12:1-9.

11. Maiden MCJ, Bygraves JA, Feil E, Morelli G, Russell JE, Urwin R, et al. Multilocus sequence typing: a portable approach to the identification of clones within populations of pathogenic microorganisms. Proc Natl Acad Sci U S A. 1998;95:3140-5.

12. Urwin R, Maiden MCJ. Multi-locus sequence typing: a tool for global epidemiology. Trends Microbiol. 2003;11:479-87. https://doi.org/10.1016/j. tim.2003.08.006.

13. Ridom SeqSphere+ - Overview. http://ridom.de/seqsphere/. Accessed 3 Feb 2020.

14. Whole genome multi locus sequence typing (wgMLST) | Applied Maths. https://www.applied-maths.com/applications/wgmlst. Accessed 3 Feb 2020.

15. Ghanem M, Wang L, Zhang Y, Edwards S, Lu A, Ley D, et al. Core genome multilocus sequence typing: a standardized approach for molecular typing of mycoplasma gallisepticum. J Clin Microbiol. 2018;56.

16. Ghanem M, El-Gazzar M. Development of mycoplasma synoviae (MS) core genome multilocus sequence typing (cgMLST) scheme. Vet Microbiol. 2018; 218:84-9.

17. Dijkman R, Feberwee A, Landman WJM. Development and evaluation of a multi-locus sequence typing scheme for Mycoplasma synoviae. Avian Pathol. 2016;9457 February:0-37.

18. Bratcher HB, Corton C, Jolley KA, Parkhill J, Maiden MCJ. A gene-by-gene population genomics platform: De novo assembly, annotation and genealogical analysis of 108 representative Neisseria meningitidis genomes. BMC Genomics. 2014;15:1-16.

19. Xiong $D$, Zhang $X, Y u$ J, Wei H. Rapid phylogenetic analysis of African swine fever virus from metagenomic sequences; 2019.

20. Volokhov DV, Grózner D, Gyuranecz M, Ferguson-Noel N, Gao Y, Bradbury JM, et al. Mycoplasma anserisalpingitidis sp. nov., isolated from European domestic geese (Anser anser domesticus) with reproductive pathology. Int J Syst Evol Microbiol. 2020.

21. Zhou Z, Alikhan NF, Sergeant MJ, Luhmann N, Vaz C, Francisco AP, et al. Grapetree: visualization of core genomic relationships among 100,000 bacterial pathogens. Genome Res. 2018;28:1395-404.

22. Kumar S, Stecher G, Li M, Knyaz C, Tamura K. MEGA X: Molecular evolutionary genetics analysis across computing platforms. Mol Biol Evol 2018;35:1547-1549.

23. Carnaccini S, Ferguson-Noel NM, Chin RP, Santoro T, Black P, Bland M, et al. A Novel Mycoplasma sp. Associated with Phallus Disease in Goose Breeders : Pathological and Bacteriological Findings A Novel Mycoplasma sp. Associated with Phallus Disease in Goose Breeders : Pathological and Bacteriological Findings. Avian Dis 2016;60:437-443.

24. Silva M, Machado MP, Silva DN, Rossi M, Moran-Gilad J, Santos S, et al. chewBBACA: a complete suite for gene-by-gene schema creation and strain identification. Microb genomics. 2018:4:1-7.

25. Delaney NF, Balenger S, Bonneaud C, Marx CJ, Hill GE, Ferguson-Noel N, et al. Ultrafast evolution and loss of CRISPRs following a host shift in a novel wildlife pathogen. Mycoplasma Gallisepticum PLoS Genet. 2012;8.

26. Woese CR, Maniloff J, Zablen LB. Phylogenetic analysis of the mycoplasmas. Proc Natl Acad Sci U S A. 1980;77:494-8.

27. FAOSTAT. http://www.fao.org/faostat/en/\#data/QL/visualize. Accessed 3 Feb 2020.

28. Babraham Bioinformatics - FastQC A Quality Control tool for High Throughput Sequence Data. https://www.bioinformatics.babraham.ac.uk/ projects/fastqc/. Accessed 3 Feb 2020. 
29. O'Connell J, Schulz-Trieglaff O, Carlson E, Hims MM, Gormley NA, Cox AJ. NxTrim: optimized trimming of Illumina mate pair reads. Bioinformatics. 2015;31:2035-7.

30. Kearse M, Moir R, Wilson A, Stones-Havas S, Cheung M, Sturrock S, et al. Geneious basic: an integrated and extendable desktop software platform for the organization and analysis of sequence data. Bioinformatics. 2012;28: 1647-9.

31. Bankevich A, Nurk S, Antipov D, Gurevich AA, Dvorkin M, Kulikov AS, et al. SPAdes: a new genome assembly algorithm and its applications to singlecell sequencing. I Comput Biol. 2012;19:455-77.

32. NCBI. Genetic codes table. https://www.ncbi.nlm.nih.gov/Taxonomy/Utils/ wprintgc.cgi\#SG4. Accessed 8 May 2020

33. Hyatt D, Chen GL, LoCascio PF, Land ML, Larimer FW, Hauser LJ. Prodigal: prokaryotic gene recognition and translation initiation site identification. BMC Bioinformatics. 2010;11.

34. Index of /blast/executables/blast+/LATEST/. ftp://ftp.ncbi.nlm.nih.gov/blast/ executables/blast+/LATEST/. Accessed 3 Feb 2020

35. Lefort V, Desper R, Gascuel O. FastME 2.0: a comprehensive, accurate, and fast distance-based phylogeny inference program. Mol Biol Evol. 2015;32: 2798-800.

36. Rasko DA, Myers GSA, Ravel J. Visualization of comparative genomic analyses by BLAST score ratio. BMC Bioinformatics. 2005:6:1-7.

37. Ribeiro-Gonçalves B, Francisco AP, Vaz C, Ramirez M, Carriço JA. PHYLOViZ online: web-based tool for visualization, phylogenetic inference, analysis and sharing of minimum spanning trees. Nucleic Acids Res. 2016:44:W246-51

38. Saitou N, Nei M. The neighbor-joining method: a new method for reconstructing phylogenetic trees. Mol Biol Evol. 1987;4:406-25.

39. DNASTAR. Bioinformatics Software for Life Science | DNASTAR | Lasergene. https://www.dnastar.com/. Accessed 8 May 2020.

\section{Publisher's Note}

Springer Nature remains neutral with regard to jurisdictional claims in published maps and institutional affiliations.

Ready to submit your research? Choose BMC and benefit from:

- fast, convenient online submission

- thorough peer review by experienced researchers in your field

- rapid publication on acceptance

- support for research data, including large and complex data types

- gold Open Access which fosters wider collaboration and increased citations

- maximum visibility for your research: over $100 \mathrm{M}$ website views per year

At $\mathrm{BMC}$, research is always in progress.

Learn more biomedcentral.com/submissions 